\title{
Depleted argon from underground sources
}

\author{
H. O. Back ${ }^{\mathrm{a} *}$, A. Alton $^{\mathrm{c}}$, F. Calaprice ${ }^{\mathrm{a}}$, C. Galbiati ${ }^{\mathrm{a}}$, A. Goretti ${ }^{\mathrm{a}}$, C. Kendziora ${ }^{\mathrm{b}}$, \\ B. Loer ${ }^{\mathrm{a}}$, D. Montanari ${ }^{\mathrm{b}}$, P. Mosteiro ${ }^{\mathrm{a}}$, and S. Pordes ${ }^{\mathrm{b}}$ \\ ${ }^{a}$ Department of Physics, Princeton University, Jadwin Hall, Princeton, NJ 08544, USA \\ ${ }^{b}$ Fermi National Accelerator Laboratory, P.O. Box 500, Batavia, IL 60510 \\ ${ }^{c}$ Augustana College, Physics Department, 2001 South Summit Ave., Sioux Fall, SD 57197
}

\begin{abstract}
Argon is a powerful scintillator and an excellent medium for detection of ionization. Its high discrimination power against minimum ionization tracks, in favor of selection of nuclear recoils, makes it an attractive medium for direct detection of WIMP dark matter. However, cosmogenic ${ }^{39} \mathrm{Ar}$ contamination in atmospheric argon limits the size of liquid argon dark matter detectors due to pile-up. The cosmic ray shielding by the earth means that Argon from deep underground is depleted in ${ }^{39} \mathrm{Ar}$. In Cortez Colorado a $\mathrm{CO}_{2}$ well has been discovered to contain approximately 500ppm of argon as a contamination in the $\mathrm{CO}_{2}$. In order to produce argon for dark matter detectors we first concentrate the argon locally to 3-5\% in an $\mathrm{Ar}, \mathrm{N}_{2}$, and He mixture, from the $\mathrm{CO}_{2}$ through chromatographic gas separation. The $\mathrm{N}_{2}$ and He will be removed by continuous cryogenic distillation in the Cryogenic Distillation Column recently built at Fermilab. In this talk we will discuss the entire extraction and purification process; with emphasis on the recent commissioning and initial performance of the cryogenic distillation column purification.
\end{abstract}

Keywords: argon; cryogenic; distillation; dark matter; scintillator; time projection chamber.

\section{Introduction}

As a strong scintillator argon is an ideal medium for Dark Matter detection. However, a limiting contamination in atmospheric argon is the beta emitter ${ }^{39} \mathrm{Ar}\left({ }^{39} \mathrm{Ar} \rightarrow{ }^{39} \mathrm{~K}+e^{-}+\overline{v_{e}}(\mathrm{Q}=565 \mathrm{keV})\right.$ [1]). It is possible to reject beta events through pulse shape analysis, but ${ }^{39} \mathrm{Ar}$ limits the size of a detector due to event pile-up. ${ }^{39} \mathrm{Ar}$ is produced in the upper atmosphere in ${ }^{40} \mathrm{Ar}(\mathrm{n}, 2 \mathrm{n})$ reactions, and is found in the atmosphere at a level of $8.1 \times 10^{-16}{ }^{39} \mathrm{Ar} /{ }^{40} \mathrm{Ar}$ [2]; corresponding to more than $1 \mathrm{~Bq} / \mathrm{kg}$ of atmospheric argon. A place to search for argon gas depleted in ${ }^{39} \mathrm{Ar}$ is deep underground where the cosmic ray flux is

\footnotetext{
* Corresponding author. Tel.: +1-630-840-2218; fax: +630-840-4263.

E-mail address: hback@fnal.gov.
} 
highly suppressed and therefore ${ }^{39} \mathrm{Ar}$ production rate is significantly reduced. We have identified a $\mathrm{CO}_{2}$ well near Cortez, Colorado that contains argon at the $600 \mathrm{ppm}$ level. The ${ }^{39} \mathrm{Ar}$ concentration in this argon has been measured to be reduced by more than a factor of 50 [3]. However, the argon must be extracted from the $\mathrm{CO}_{2}$ and further purified in order to be used in a detector.

\section{Chromatographic Gas Separation}

It is possible to trap specific gases on an absorptive medium, while allowing other gases to pass [4]. The absorption efficiency is directly proportional to the gas pressure, and this property can be exploited to create very efficient absorption devices, and to regenerate the absorptive medium. Outside of Cortez Colorado we have built a vacuum-pressure swing absorption (VPSA) unit to extract the depleted argon from a $\mathrm{CO}_{2}$ well. $\mathrm{CO}_{2}, \mathrm{O}_{2}$, and other unwanted gases are trapped on Zeolite with high efficiency under pressure [5]. Eventually the Zeolite will become saturated with the unwanted gases, and they are then able to pass through the column. Therefore, the flow is stopped through the column, and a vacuum pump is applied to the system to force the Zeolite to release the contaminants. By using two columns we can create a continuous production by absorbing on one column, while the other column is being regenerated. Figure 1a shows an illustration of the two column VPSA principle. Our unit consists of 2 stages, of 2 columns each (figure 2a). The first stage traps $\mathrm{CO}_{2}, \mathrm{O}_{2}$, and other unwanted gases, and while still under pressure the second stage continues to remove these gases as well as some of the $\mathrm{N}_{2}$.

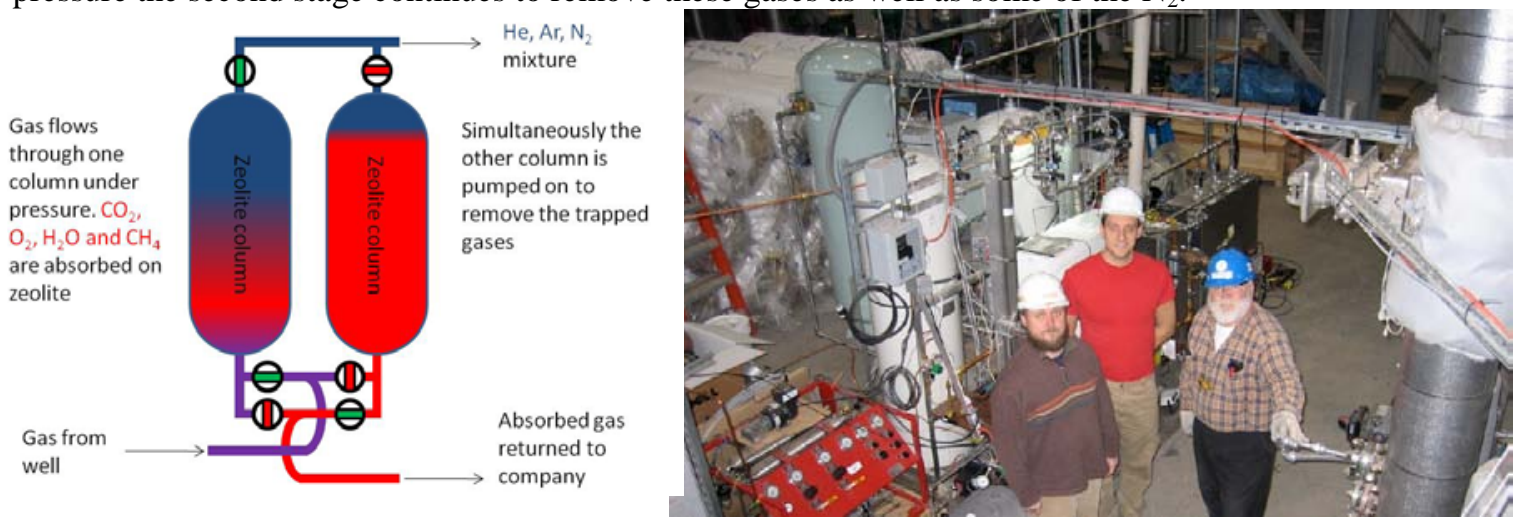

Figure 1.(a) Illustration of the VPSA gas extraction principle; (b) VPSA system in Cortez, CO

The VPSA system in Cortez, CO, has been operating since February 2010. It effectively concentrates argon, but there are some gases that are currently irreducible. Helium cannot be separated by using the VPSA, and nitrogen is not effectively removed by the columns. In 2011 we were able to reduce the nitrogen and increase the argon concentration, but this also had the effect of increasing the total amount of helium. In Table 1 we see what the gas fractions are for the gas coming from the well and what the fractions are after the VPSA unit during the 2010 running, and the 2011 running. At peak performance when the plant is in production mode it produces argon at a rate of approximately $0.5 \mathrm{~kg} / \mathrm{day}$. As of June 2011 we had collected approximately $44 \mathrm{~kg}$ of argon in pressurized cylinders. 
Table 1. Composition of the well gas before and after the VPSA extraction. The fraction of argon is increased significantly

\begin{tabular}{llll}
\hline Gas Type & Concentration from well & $\begin{array}{l}\text { 2010 concentration after } \\
\text { VPSA extraction }\end{array}$ & $\begin{array}{l}\text { 2011 concentration after } \\
\text { VPSA extraction }\end{array}$ \\
\hline Carbon Dioxide & $96 \%$ & Below sensitivity & Below sensitivity \\
Nitrogen & $2.4 \%$ & $70 \%$ & $40 \%$ \\
Methane & $5,700 \mathrm{ppm}$ & Below sensitivity & Below sensitivity \\
Helium & $4,300 \mathrm{ppm}$ & $27.5 \%$ & $55 \%$ \\
Other hydrocarbons & $2,100 \mathrm{ppm}$ & Below sensitivity & Below sensitivity \\
Water & $1,000 \mathrm{ppm}$ & Below sensitivity & Below sensitivity \\
Argon & $600 \mathrm{ppm}$ & $2.5 \%$ & $5 \%$ \\
Oxygen & Below sensitivity & Below sensitivity & Below sensitivity \\
\hline
\end{tabular}

\section{Continuous Distillation}

It is well understood that the difference in boiling points of the constituents in a multi component liquid allows for separation through distillation [6]. It is possible to perform distillation continuously in a column packed with a high surface area material, and a temperature gradient [7]. The liquid will boil and the gas condenses continuously on the packing material. Gases rise and recondense, while liquids sink and reboil. The component with the lower boiling point rises preferentially and the component with the higher boiling point falls to the lower volume. By maintaining a temperature gradient and a constant flow of liquid into the column, an equilibrium is established, and very pure material can be extracted continuously from the column. Figure 2 shows an illustration of this process.

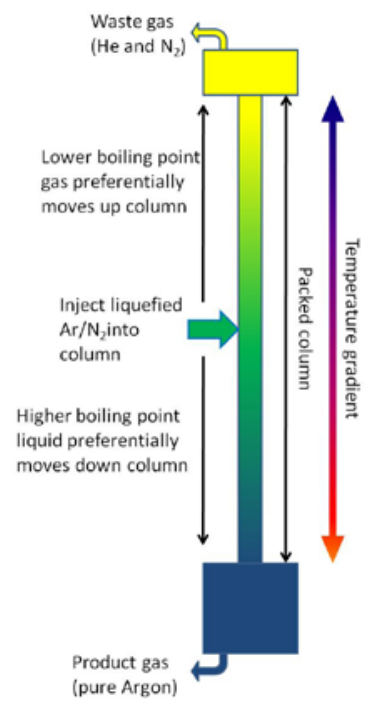

Figure 2. Illustration of the continuous distillation in a packed column process principle

\subsection{Fermilab Cryogenic Distillation Column}

At Fermilab a cryogenic distillation column has been constructed for the purification of the gas extracted from the $\mathrm{CO}_{2}$ wells in Cortez, $\mathrm{CO}$ (Figure 3). The column is $320 \mathrm{~cm}$ tall and $2 \mathrm{~cm}$ in diameter, and is filled with a custom packing material (Figure 4), which makes this column equivalent to 40 theoretical stages. A 600 watt cryocooler regulated with a 600 watt heater is used to liquefy the $\mathrm{N}_{2}$ and $\mathrm{Ar}$ 
gases, which are then injected in the column. The helium is not liquefied and is passed through the column to the waste. The column is cooled with a 600 watt cryocooler, and the temperature gradient is maintained with heaters at the top and bottom of the column. Gas flow control, temperature monitoring, and temperature control are all performed with a Labview based central control system. The input, waste, and product gas streams are all monitored with a Universal Gas Analyzer (UGA) to monitor the distillation column performance. In its final configuration the Distillation Column will be able to produce $99.9999 \%$ pure argon at a rate of $5-10 \mathrm{~kg} /$ day.

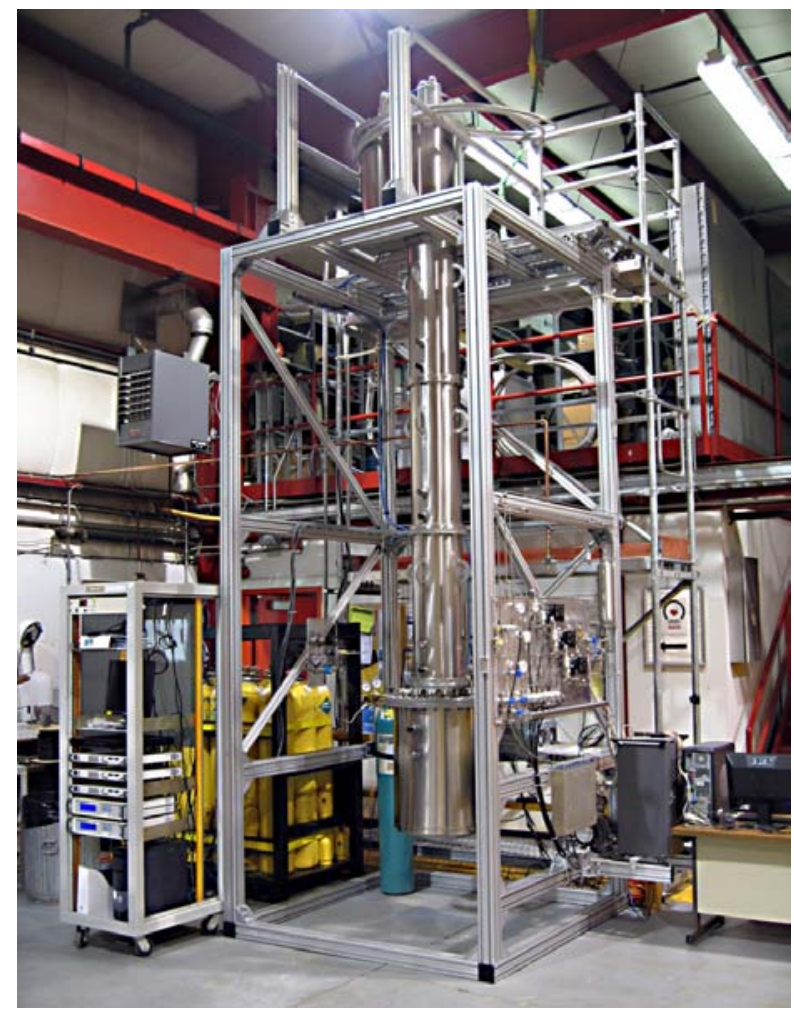

Figure 3. Cryogenic Distillation Column at Fermi National Accelerator Laboratory

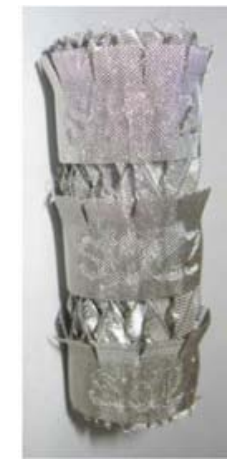

Figure 4.Custom packing material in the distillation column 


\subsection{Fermilab Cryogenic Distillation Column Commissioning}

In March 2011 the Distillation Column was run for the first time using a feed gas mixture with a composition identical to the gas extracted by the VPSA during 2010 (Table 1). This initial commissioning run had three goals:

- Condense all argon from the mixed feed gas stream.

- Show that continuous distillation is possible.

- Produce a batch of pure argon.

Waste gas was expelled during this commissioning, and the product gas was collected as a liquid in the reboiler volume of the distillation column.

To monitor the performance of the distillation column we primarily monitored the gas exiting the waste with the UGA. The feed gas was a known mixture that was certified by the supplier, and therefore monitoring was not required. However, since a UGA cannot provide the exact composition of unknown gases, we compared the UGA measurements of the feed, waste and product streams to make relative measurements.

In our first test we tuned the temperature of the column until we were collecting $100 \%$ of the argon. At this level we are also collecting a fraction of the nitrogen. Figure 5 is a UGA scan of the waste stream were we can see that after 5 hours the amount of argon in the waste stream is zero, meaning that all the argon is being collected in the distillation column.

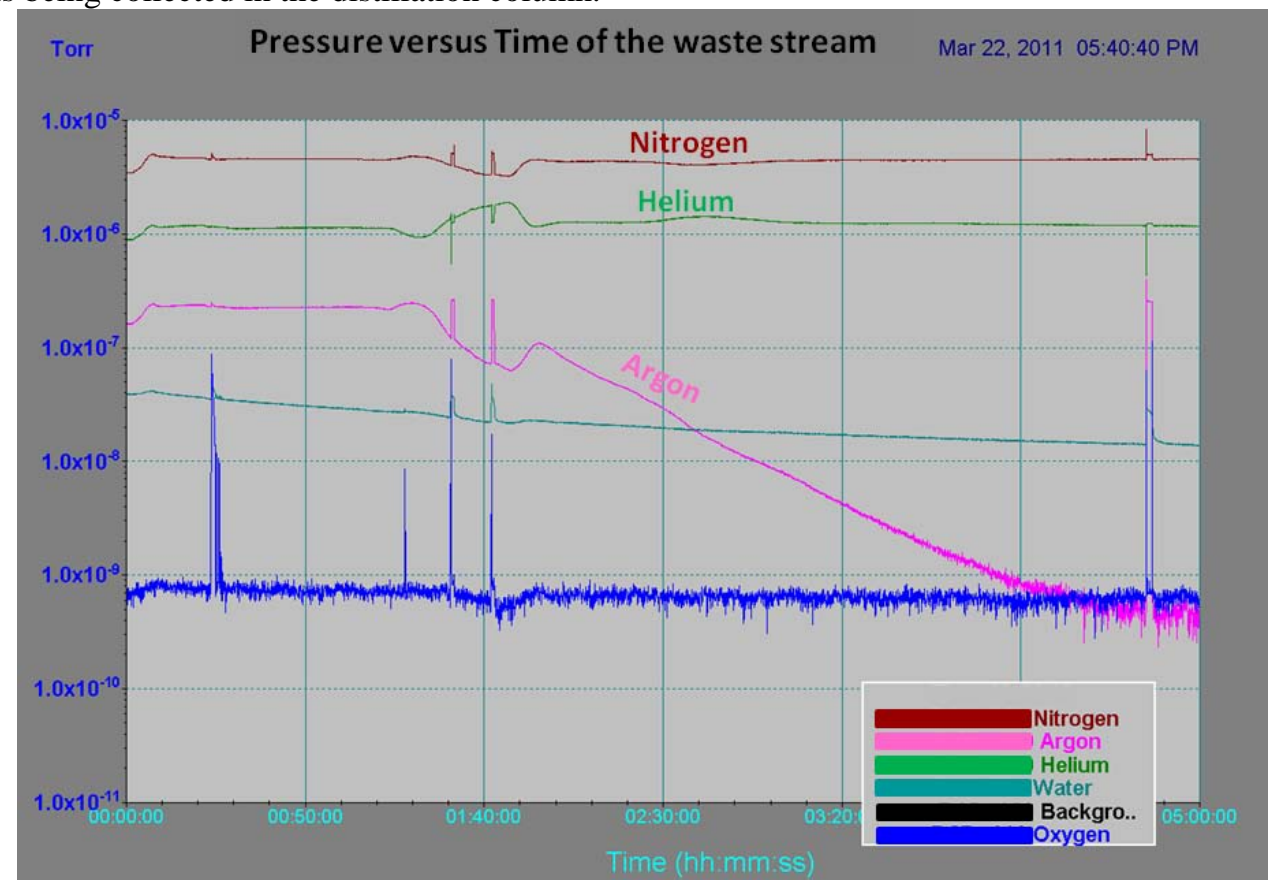

Figure 5. Gas composition of the waste stream from the distillation column during the initial cool down and temperature tuning

Although the UGA cannot make a quantitative measurement of the gas composition, we are in a unique position to make some relative measurements. The helium cannot be collected by the Distillation Column, and therefore any helium that enters the input must leave the waste. This allows us to compare the amount of argon and nitrogen relative to the helium in the feed gas and waste streams. This method was used to tune the temperature of the column until continuous distillation was established. Figure 6 
shows the gas composition of the feed gas and waste gas during temperature tuning. The ratio of argon and nitrogen to helium for the feed and waste gases is shown in Table 2. From these ratios we can see that more nitrogen is leaving the distillation column through the waste stream than we are feeding into the input. Conversely there is much less argon leaving the distillation column than we are feeding into the input; proving that a continuous distillation state has been achieved in the Distillation Column.

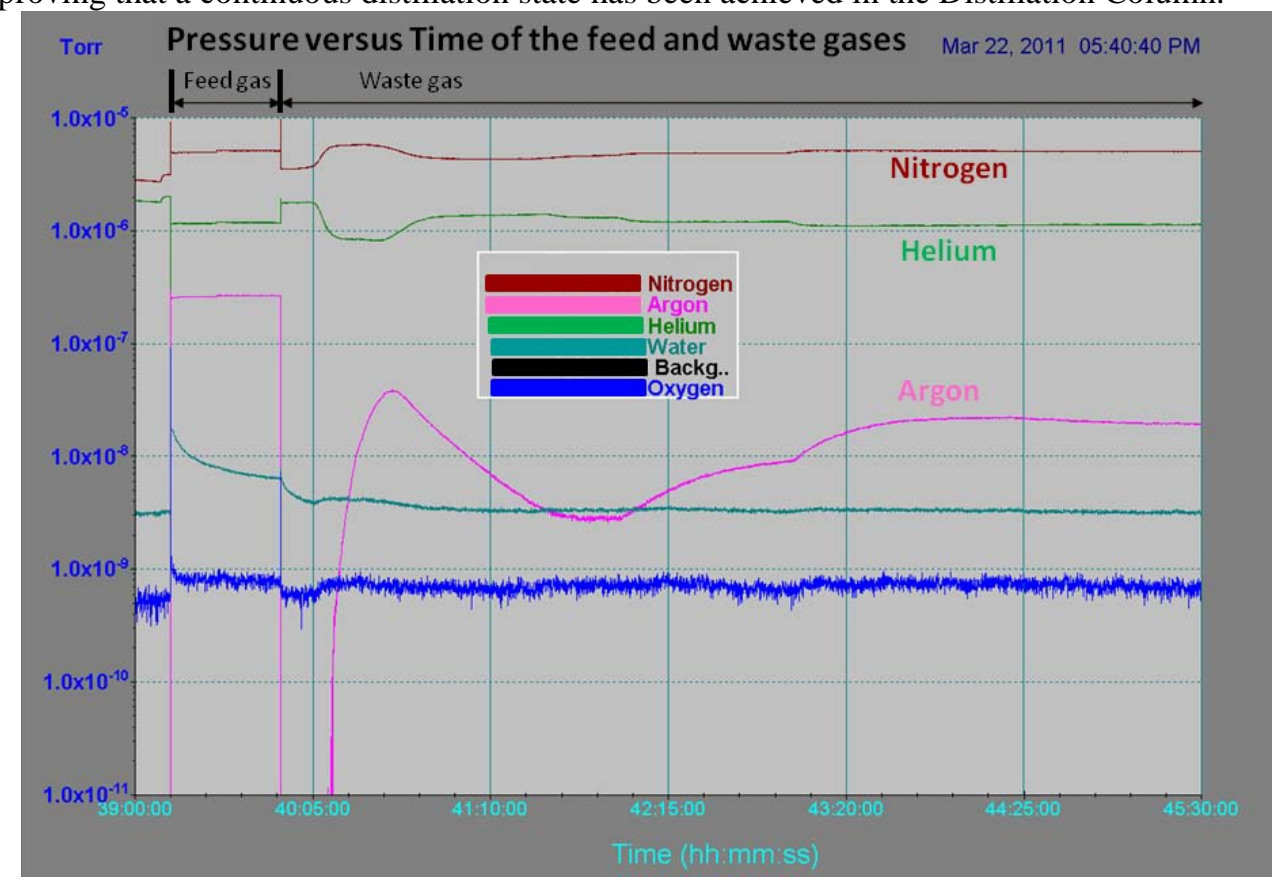

Figure 6. Waste and feed gas composition during temperature tuning for continuous distillation

Table 2. Gas composition ratios of the feed gas and the waste gas during continuous distillation.

\begin{tabular}{ccc}
\hline Gas component ratios & Feed Gas & Waste Gas \\
\hline $\mathrm{N}_{2} / \mathrm{He}$ & 4.23 & 4.43 \\
$\mathrm{Ar} / \mathrm{He}$ & 0.22 & 0.02 \\
\hline
\end{tabular}

The distillation column was run in the mode of collecting argon and nitrogen for a long time before the continuous distillation was established. He had collected so much nitrogen that making a pure batch of Argon through continuous distillation was prohibited by time constraints. Therefore, the feed gas was turned off, the product gas was directed back into the input, and batch purification was started. In this configuration there is no feed gas to monitor, but we can monitor the product gas as well as the waste gas. The monitoring of the gas was switched between the product and waste gas frequently to continuously monitor both streams. In Figure 7 the nitrogen concentration in the product stream slowly falls while the argon concentration increases, until eventually argon is observed in the waste stream and within an hour the nitrogen concentration in both the waste and input streams falls. 


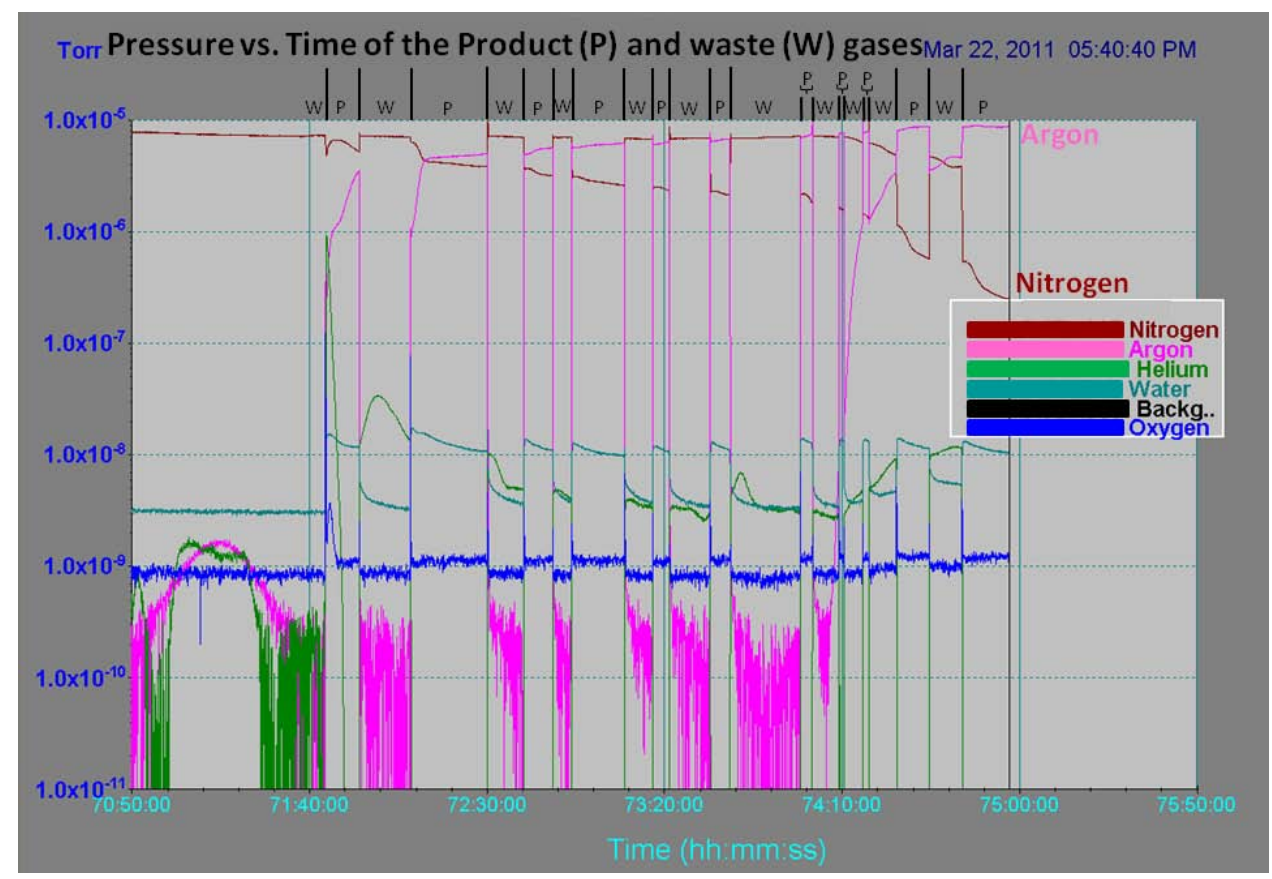

Figure 7. Product and waste gas compositions during batch distillation. W denotes waste and P denotes product

When the test was stopped the Ar: $\mathrm{N}_{2}$ ratio was 33:1, corresponding to approximately $97 \%$ pure argon, the other $3 \%$ being nitrogen. The input Ar: $\mathrm{N}_{2}$ ratio was 1:28 concluding that the distillation column was able to reduce the amount of nitrogen by more than a factor of 1000 .

\section{Conclusions}

From a concentration of 600ppm of Ar in the well gas in Colorado we have been able to produce 97\% pure argon through chromatographic gas separation in Colorado with the VPSA plant and distillation with the cryogenic distillation column at Fermilab. In the near future the performance of the distillation column will be studied through further commissioning, and with recent upgrades the throughput is a step closer to the $10 \mathrm{~kg} /$ day goal, and a purity of $<10 \mathrm{ppm}$ nitrogen in the argon is expected to be achievable.

\section{Acknowledgments}

We thank Kinder Morgan $\mathrm{CO}_{2}$ Company for hosting us in their facilities in Cortez for the argon extraction part of this project.

\section{References}

[1] R. B. Firestone, Table of Isotopes Eighth Edition (Wiley-Interscience, 1996), p. 807

[2] H. H. Loosli, Earth Planet. Sci. Lett. 63, 51-62 (1983)

[3] A. Wright et al. (DarkSide Collaboration), Submitted to Proceedings of the 2011 Meeting of the Division of Particles and Fields of the American Physical Society, September 2011. arXiv:1109.2979., 
[4] A.Pocar, Ph.D. Thesis, Princeton University, 2003

[5] D. Acosta-Kane et al., Nucl. Instr. and Meth. A 587 (2008) 46

[6] A. L. Arey, Elementary Chemistry for High Schools and Academies (The MacMillan Company, New York, 1900), p. 204

[7] W. L. McCabe and J. C. Smith, Unit Operations of Chemical Engineering, (3rd Edition, McGraw-Hill 1976); Perry's Chemical Engineers' Handbook, (7th Edition, McGraw-Hill 1997); Ivar J. Halvorsen and Sigurd Skogestad, Encyclopedia of Separation Science, (Chapter 2, Academic Press Ltd. 1999) 